University of Wollongong

Research Online

Faculty of Social Sciences - Papers (Archive) Faculty of Arts, Social Sciences \& Humanities

$1-1-2017$

Cognitive remediation improves executive functions, self-regulation and quality of life in residents of a substance use disorder therapeutic community

Ely Marceau

University of Wollongong, emm993@uowmail.edu.au

Jamie Berry

Advanced Neuropsychological Treatment Services

Jo Lunn

We Help Ourselves

Peter James Kelly

University of Wollongong, pkelly@uow.edu.au

Nadia Solowij

University of Wollongong, nadia@uow.edu.au

Follow this and additional works at: https://ro.uow.edu.au/sspapers

Part of the Education Commons, and the Social and Behavioral Sciences Commons

Research Online is the open access institutional repository for the University of Wollongong. For further information contact the UOW Library: research-pubs@uow.edu.au 


\title{
Cognitive remediation improves executive functions, self-regulation and quality of life in residents of a substance use disorder therapeutic community
}

\author{
Abstract \\ Background Executive dysfunction is common in substance use disorder (SUD) populations and hinders \\ treatment. We previously found that $50 \%$ of residents in SUD therapeutic communities had been \\ hospitalized for head injuries; this was a significant determinant of cognitive impairment. The current \\ study aimed to establish whether cognitive remediation improves executive functions (EFs) and self- \\ regulation in an ecologically valid sample of female residents attending SUD therapeutic community \\ treatment, including those with past head injuries and psychiatric comorbidities. Methods Controlled \\ sequential groups design with residents ( $N=33$, all female) receiving treatment as usual (TAU). The \\ intervention group $(n=16)$ completed four weeks of cognitive remediation (CR) and the control, TAU only \\ $(n=17)$. Outcome measures assessed pre- and post-intervention included both performance- and \\ inventory-based measures of EFs, and self-reported self-regulation and quality of life. Results CR relative \\ to TAU significantly improved performance-based assessment of inhibition (Color-Word Interference Test; \\ $F=4.29, p=0.047$ ), inventory-based assessment of EFs (Behavior Rating Inventory of Executive Function \\ $¿$ Adult Version: Global Executive Composite; $F=6.38, p=0.017$ ), impulsivity (Barratt Impulsiveness \\ Scale; $F=4.61, p=0.040$ ), self-control (Brief Self-Control Scale; $F=5.53, p=0.026$ ), and quality of life \\ (Quality of Life Enjoyment and Satisfaction Questionnaire ¿ Short Form; F = 7.68, $p=0.010$ ). Conclusions \\ Findings suggest that $\mathrm{CR}$ improves EFs in a heterogeneous sample of female residents in therapeutic \\ community SUD treatment. Future research may explore the possibility of tailoring CR interventions for \\ various SUD subgroups.

\section{Disciplines} \\ Education | Social and Behavioral Sciences

\section{Publication Details} \\ Marceau, E., Berry, J., Lunn, J., Kelly, P. \& Solowij, N. (2017). Cognitive remediation improves executive \\ functions, self-regulation and quality of life in residents of a substance use disorder therapeutic \\ community. Drug and Alcohol Dependence, 178 150-158.
}


Drug and Alcohol Dependence 2017, 178, 150-158.

Cognitive remediation improves executive functions, self-regulation and quality of life in residents of a substance use disorder therapeutic community

Ely M. Marceau ${ }^{\mathrm{a}}$, Jamie Berry ${ }^{\mathrm{b}}$, Joanne Lunn ${ }^{\mathrm{c}}$, Peter J. Kelly ${ }^{\mathrm{a}}$, and Nadia Solowij ${ }^{\mathrm{a} *}$

${ }^{a}$ School of Psychology and Illawarra Health and Medical Research Institute,

University of Wollongong, Northfields Ave Wollongong NSW 2522 Australia

${ }^{\mathrm{b}}$ Advanced Neuropsychological Treatment Services, PO Box 4070 Strathfield South NSW 2136 Australia

${ }^{\mathrm{c}}$ We Help Ourselves (WHOs), Building 128, Church St Lilyfield NSW 2040

Australia

* Corresponding Author at:

School of Psychology, University of Wollongong

Northfields Ave Wollongong NSW 2522 Australia

Tel: +61242213732, Fax: +61242214163, E-mail: nadia@uow.edu.au 


\begin{abstract}
Background

Executive dysfunction is common in substance use disorder (SUD) populations and hinders treatment. We previously found that $50 \%$ of residents in SUD therapeutic communities had been hospitalized for head injuries; this was a significant determinant of cognitive impairment. The current study aimed to establish whether cognitive remediation improves executive functions (EFs) and self-regulation in an ecologically valid sample of female residents attending SUD therapeutic community treatment, including those with past head injuries and psychiatric comorbidities.
\end{abstract} Methods

Controlled sequential groups design with residents $(N=33$, all female) receiving treatment as usual (TAU). The intervention group $(n=16)$ completed four weeks of cognitive remediation (CR) and the control, TAU only $(n=17)$. Outcome measures assessed pre- and post-intervention included both performance- and inventory-based measures of EFs, and self-reported self-regulation and quality of life.

Results

CR relative to TAU significantly improved performance-based assessment of inhibition (Color-Word Interference Test; $F=4.29, p=0.047$ ), inventory-based assessment of EFs (Behavior Rating Inventory of Executive Function - Adult Version: Global Executive Composite; $F=6.38, p=0.017$ ), impulsivity (Barratt Impulsiveness Scale; $F=4.61, p=0.040$ ), self-control (Brief Self-Control Scale; $F=5.53, p=$ 0.026) and quality of life (Quality of Life Enjoyment and Satisfaction Questionnaire Short Form; $F=7.68, p=0.010)$.

\title{
Conclusions
}

Findings suggest that CR improves EFs in a heterogeneous sample of female residents in therapeutic community SUD treatment. Future research may explore the possibility of tailoring CR interventions for various SUD subgroups.

\section{Keywords:}

Cognitive Remediation; Executive Functions; Self-Regulation; Substance Use Disorder; Therapeutic Community 


\section{Introduction}

Self-regulation is a protective factor in ameliorating many social and mental health problems and is understood as the capacity "to make plans, choose from alternatives, control impulses, inhibit unwanted thoughts and regulate social behavior" (Heatherton \& Wagner, 2011, p. 132). Individuals in residential rehabilitation treatment for substance use disorders (SUDs) must possess some capacity for selfregulation in order to meaningfully engage in treatment and ultimately change their behavior. This is particularly true of Therapeutic Community treatment (Vanderplasschen et al., 2013), which entails significant social participation and shared responsibility for activities of daily life.

Cognitive deficits are one of the four biggest risk factors for dropout from SUD treatment (Brorson et al., 2013), and executive function (EF) impairment is commonly observed in individuals experiencing SUDs (Fernández-Serrano et al., 2010; Hester et al., 2010). Diverse definitions of EFs exist (Friedman \& Miyake, 2016) but they are broadly understood as "those capacities that enable a person to engage successfully in independent, purposive, self-directed, and self-serving behavior" (Lezak et al., 2012, p. 37). An influential threefold model of EFs includes ‘working memory', ‘inhibition', and 'shifting' (Miyake et al., 2000). Working memory refers to the capacity to monitor and alter information held in mind temporarily, inhibition involves overriding an unwanted distraction to maintain taskfocus, and shifting pertains to flexibly switching attention between tasks or mental sets (Hofmann et al., 2012). These basic EFs are intricately linked to and may subserve effective self-regulation (Hofmann et al., 2012).

Individuals in residential treatment for SUDs often have psychiatric and medical comorbidities, including history of head injury. For example, we previously found that $67.2 \%$ of residents in SUD Therapeutic Community treatment $(70.3 \%$ 
male) had sustained one or more head injuries, while $50 \%$ required hospitalisation following a head injury (Marceau et al., 2016). History of head injury was a significant determinant of cognitive impairment and was associated with higher levels of psychological distress. Psychiatric comorbidities are also frequently observed in SUD populations and add further complexity to the process of addiction recovery (Baingana et al., 2015). Personality disorders are particularly prevalent (e.g. Pennay et al., 2011) and are not only associated with global neurocognitive and specific EF deficits, (Fertuck et al., 2006; Ruocco \& Carcone, 2016; Unoka \& Richman, 2016), but also with dropout from SUD treatment (Brorson et al., 2013).

Given the high rates of head injury and psychiatric comorbidities in SUD populations, and the positive relationship of these variables with cognitive impairment, the application of evidence-based neuropsychological interventions designed for use in both brain injury (usually referred to as cognitive rehabilitation) and mental health (usually referred to as cognitive remediation) populations might be expected to result in reductions of cognitive impairment, and potentially lead to better SUD treatment outcomes. It has been recommended that these interventions be adapted for use in SUD treatment populations (Bates et al., 2013a) and there have been a number of attempts at this to date (e.g., Alfonso et al., 2011; Bickel et al., 2011; Houben et al., 2011; Valls-Serrano et al., 2016a).

Neuropsychological interventions for SUD populations have tended to adopt either a drill and practice (e.g., Houben et al., 2011) or strategy-based (e.g., VallsSerrano et al., 2016a) approach. To our knowledge, there are no published studies incorporating a combined approach that utilises both domains. The cognitive remediation literature within psychiatry has suggested that whilst drill and practice approaches (e.g., computerized cognitive training) may lead to greater gains on 
cognitive test scores, strategy-based training (e.g., instruction in specific strategy use) leads to greater functional outcomes for individuals with schizophrenia (Paquin et al., 2014; Wykes et al., 2011). Combining approaches in an SUD population may result in greater gains across a range of measures. This study aimed to determine the effectiveness of a cognitive remediation intervention that incorporated both drill and practice and strategy-based training for a treatment seeking SUD population, inclusive of those with psychiatric and head injury comorbidities.

Assessment of EFs may be performance- (i.e., assessing performance on working memory, inhibition, and shifting tasks) or inventory-based (i.e., based on self-reports of executive functioning). Whilst performance-based measures of EFs are sensitive to brain impairment that implicates the frontal lobes (Jurado \& Roselli, 2007), some may demonstrate limited ecological validity and may not capture problems with everyday functioning as well as inventory-based measures (Isquith et al., 2013). Inventory- and performance-based measures of EFs are minimally correlated and may assess distinct components of EFs that contribute independently to clinical problems (Toplak et al., 2013). For example, to determine the relative sensitivities of performance- and inventory-based EFs measures in an SUD treatment population, Hagen and colleagues (2016) showed that inventory-based assessment using the Behavior Rating Inventory of Executive Function - Adult Version (BRIEFA; Roth et al., 2005) better distinguished polysubstance users from controls and was more strongly associated with real-world social adjustment outcomes compared to performance-based measures, which included the Iowa Gambling Task (Bechara et al., 1994), the Trail-Making Test (Strauss et al., 2006), and the Stroop test (Golden, 1978). In light of these findings and to provide a more comprehensive assessment of EFs, the current study included both performance- and inventory-based measures. 
The current study aimed to examine the effectiveness of cognitive remediation vs. treatment-as-usual (TAU) in: (1) improving performance- and inventory-based measures of EFs and (2) improving self-report measures of self-regulation. Quality of life was included as a secondary clinical outcome measure, as it plays an important role in sustained remission from SUDs (Laudet et al., 2009). We hypothesized that cognitive remediation would be effective in improving EFs, self-regulation, and quality of life.

\section{Methods}

\subsection{Participants}

Fifty participants were recruited from a women's residential treatment facility in Sydney run by We Help Ourselves (WHOs) - a large provider of residential SUD rehabilitation in Australia, utilising the Therapeutic Community model of treatment. Inclusion criteria for the study were: (i) diagnosis of substance abuse/dependence, based on DSM-IV-TR criteria, assessed using the Mini-International Neuropsychiatric Interview (MINI-Plus; Sheehan et al., 1998), (ii) a minimum abstinence period of 7 days (with confirmation of detoxification a prerequisite of entry to treatment), (iii) absence of any neurological, infectious, or other disease affecting the central nervous system (e.g., epileptic seizures, stroke, brain tumour, meningitis, encephalitis, multiple sclerosis, HIV positive), and (iv) English as native language. A condition of staying at the residential facility is that participants remain abstinent from substances of abuse and this is monitored through routine urinalysis (random resident checks occurring several times per week) and 24-hour observation from experienced staff and co-residents. 


\subsection{Diagnostic and clinical assessment}

Diagnostic and clinical assessment at baseline included the following: Psychiatric comorbidities (DSM-IV-TR) were assessed using the MINI-Plus and Standardised Assessment of Personality - Abbreviated Scale (Moran et al., 2003), as shown to be appropriate for SUD populations (Gonzalez, 2014; Hesse \& Moran, 2010; Hesse et al., 2008). Questions were adapted from the Addiction Severity Index - Fifth Edition (ASI; McLellan et al., 1992) to assess lifetime substance use history. Additionally, a brief semi-structured interview was used to assess history of head injury. The Test of Premorbid Functioning (TOPF; Pearson Assessment, 2009) was used to estimate overall level of intellect.

\subsection{Outcome measures}

\subsubsection{Executive functions - performance-based}

2.3.1.1. Working memory: Working Memory Index (WMI; Wechsler Adult Intelligence Scale, fourth edition: WAIS-IV; Wechsler, 2008). The WMI of the WAIS-IV assesses components of working memory and is comprised of 2 subtests, which were administered according to standard instructions. The digit span subtest requires participants to recall various sequences of numbers (forward, backward, and in sequence) and the arithmetic subtest involves participants solving numerical problems within 30 seconds, after they have been read aloud by the examiner. The subtest scores were summed to yield a total score, which was then scaled to provide an index score, as per standardized scoring instructions.

\subsubsection{Inhibition: Color-Word Interference Test (Delis-Kaplan Executive Function} System: D-KEFS; Delis et al., 2001). This subtest of the D-KEFS assesses response inhibition and provides an auxiliary measure of shifting. Participants are instructed to 
read the items presented in each of four conditions as quickly and accurately as possible. Performance is measured in time (seconds). The first condition presents patches of colours and requires participants to name the colours. The second condition presents the words "red", "blue", and "green" and requires participants to read the words. The third condition presents words printed in incongruent colours and requires the participant to ignore the word and say the colour. The fourth condition presents words printed in incongruent colours and requires the participant to switch between two rules: (a) ignore the word and say the colour; and (b) ignore the colour and say the word. Outcome variables were contrast scaled scores of inhibition (condition 3 scaled score minus condition 1 scaled score) and inhibition/shifting (condition 4 scaled score minus condition 1 scaled score).

2.3.1.3. Shifting: Trail-Making Test (TMT; Strauss et al., 2006). This test provides a measure of shifting, with Part A assessing simple psychomotor ability and Part B assessing psychomotor ability and shifting. In part A, participants connect 25 numbered circles in ascending order. In part B, 13 numbers and 12 letters have to be alternately connected in their numerical and alphabetical order. Participants are notified of any errors immediately and must correct them without assistance. The outcome variable was the difference in time (seconds) to complete part B versus part A (time B minus time A), which specifically reflects shifting.

\subsubsection{Executive functions - inventory-based}

Behavior Rating Inventory of Executive Function - Adult Version (BRIEF-A; Roth et al., 2005). The BRIEF-A is a 75-item self-report questionnaire consisting of nine subscales. Participants are instructed to answer each question by selecting "never", "sometimes", or "often", in relation to whether they have had problems with any of the listed behaviors in the past month. Example items include: "I have trouble getting 
ready for the day"; "I have trouble coming up with ideas for what to do with my free time". The Global Executive Composite (GEC) provides an overall summary score. The Behavioral Regulation Index (BRI) includes the subscales Inhibit; Shift; Emotional Control; Self-Monitor. The Metacognition Index (MI) includes subscales Initiate; Working Memory; Plan/Organize; Task Monitor; Organisation of Materials. Elevated scores indicate executive dysfunction. Scores on the GEC, BRI, and MI subscales were used as outcome variables in the analyses.

\subsubsection{Self-regulation}

\subsubsection{Barratt Impulsiveness Scale - Version 11 (BIS-11; Patton et al., 1995). The}

BIS-11 is a 30-item self-report questionnaire that assesses trait and behavioral aspects of the construct of impulsivity. There are six first-order factors: Attention, Cognitive Instability, Motor, Perseverance, Self-Control, and Cognitive Complexity. These firstorder factor scores were summed to yield a total score, which was used as the outcome variable.

2.3.3.2. Brief Self-Control Scale (BSCS; Tangney et al., 2004). The BSCS is a 13-item self-report questionnaire that assesses individual differences in the construct of selfcontrol. The outcome variable was total score.

2.3.3.3. Difficulties in Emotion Regulation Scale (DERS; Gratz \& Roemer, 2004). The DERS is a 41-item self-report questionnaire that assesses clinically relevant difficulties in emotion regulation. There are six subscales (Nonacceptance of Emotional Responses, Difficulties Engaging in Goal-Directed Behavior, Impulse Control Difficulties, Lack of Emotional Awareness, Limited Access to Emotion Regulation Strategies, and Lack of Emotional Clarity) contributing to a total score, which was used as the outcome variable. 
2.3.3.4. Penn Alcohol Craving Scale (PACS; Flannery et al., 1999). The PACS is a 5item self-report questionnaire that assesses level of cravings for alcohol and other drugs. Participants were asked to respond to all items in relation to their primary substance of misuse. The outcome variable was total score.

2.3.4. Quality of Life Enjoyment and Satisfaction Questionnaire - short form (Q-LESQ-SF; Endicott et al., 1993). The Q-LES-Q-SF is a 16-item self-report questionnaire that assesses quality of life across areas of daily functioning. Total score was used as the outcome variable.

\subsection{Design and procedures}

The study employed a controlled sequential groups design and was a naturalistic study, capturing a snapshot of residents in treatment at the time of recruitment. This design was chosen due to resource constraints and logistic difficulty of having the small sample of residents in treatment at any one time being allocated to differing treatment regimes. All residents in the treatment facility were invited to participate in the study and the response rate was $96 \%$. Those who met inclusion criteria and provided consent took part in either: (a) a cognitive remediation (CR; $n=$ 23) group program or (b) treatment as usual (TAU; $n=27)$. The CR group were recruited first followed by the TAU group, allowing sufficient time for a new cohort of residents to become available. Unexpectedly, the CR group had spent more time in treatment prior to baseline assessment $(\mathrm{Mdn}=67$ days $)$ than the TAU group $(\mathrm{Mdn}=$ 25 days).

Both groups engaged in the usual Therapeutic Community model of treatment (Vanderplasschen et al., 2013). In addition to this, the CR group attended a total of 12 x 2-hour group sessions across 4 weeks (3 sessions per week held on Mondays, 
Wednesdays, and Fridays). All participants in the CR group attended these sessions at the same time, with all sessions facilitated by the second author (JB) and cofacilitated by the first author (EM). The first hour of each session was dedicated to strategy training, which included traditional instructional pedagogical approaches, modelling, exercises to demonstrate concepts, and role-plays. The second hour included group computerized cognitive training using the Lumosity application (Lumosity, 2016) on iPads. Although Lumosity has not previously been used in SUD populations, it has been utilized across a broad range of other clinical populations (e.g., multiple sclerosis (Charvet et al., 2015), childhood cancer-related brain injury (Kesler et al., 2011) and mild cognitive impairment (Dannhauser et al., 2014)) and was chosen for its web-based platform and ease of access. Participants played specific games during which they were instructed to use and practice certain strategies that linked to the strategy-based learning in the previous hour. They were asked to share other strategies they may have found useful during the cognitive exercises and this was discussed and sometimes integrated with the learning material by the facilitators. The amount of time spent training on each game was held constant, rather than the number of trials of each game. This allowed participants to progress through the exercises at their own pace. The CR intervention was developed with a strong emphasis on the training of EFs and self-regulation in view of the finding that EFs are particularly impaired in an SUD treatment population. The intervention incorporated elements from well researched CR interventions designed for an acquired brain injury (ABI) population, including self-alert training (O'Connell et al., 2008; Robertson et al., 1995), goal management training (Levine et al., 2011), time pressure management (Fasotti et al., 2000), and multifaceted treatment of executive dysfunction (Spikman et al., 2010). Other relevant evidence-based interventions originally designed for a non- 
ABI population were also incorporated, such as mental contrasting and implementation intentions (Duckworth et al., 2011). Barkley's (2001; 2012) evolutionary model of EFs was used to structure a large component of the program. The modules covered the following topics: levels of brain functioning, attention, learning and memory, executive functions, self-awareness, inhibition, visual and verbal working memory, emotion regulation, decision-making and problem-solving. The facilitators followed a manual (available on request) to ensure treatment consistency.

The CR group completed baseline assessments in the week prior to the commencement of the intervention and post-intervention assessments in the week following its completion. Similarly, the TAU group completed baseline assessments followed by a period of approximately 4 weeks and were reassessed during the subsequent week. There was a 5-day difference between groups in the interval between baseline and post-intervention assessments (CR: Mdn = 34 days; TAU: Mdn $=29$ days). The final sample size, accounting for treatment dropout, was 33: $n=16$ (CR group) and $n=17$ (TAU group). During the study period, the rates of unplanned discharges from the residential program did not significantly differ between groups $\left(\mathrm{CR}=30 \%, \mathrm{TAU}=37 \%, X^{2}=0.24, p=0.62\right)$ and are comparable to those observed in SUD Therapeutic Community treatment (Darke et al., 2012).

\subsection{Statistical methods}

All statistical analyses were performed using IBM Statistical Package for the Social Sciences (SPSS version 21; IBM Corp, 2012). Standardized residuals were assessed to detect possible outliers (values greater than \pm 3 ). Two outliers were found in the Inhibition condition of the Color-Word Interference Test and one was found in 
the Trail Making Test B-A. With exclusion of these outliers from analyses, the pattern of results did not change and therefore outliers were retained. One missing value was detected in the TAU group (PACS) and three were detected in the CR group (BSCS; DERS; Q-LES-Q-SF).

Socio-demographic characteristics of the CR vs. TAU groups were compared using Student's $t$-test and Mann-Whitney $U$ tests as a nonparametric alternative. Chisquare and Fisher's exact tests were used to compare percentage variables.

Despite significant differences in treatment duration and days from baseline to post-intervention between groups, these variables were not included as covariates in subsequent analyses. This is based on the recommendations of Kraemer (2015) that covariates should be selected a priori and be as few in number as possible, with a subsequent option of examining moderators of treatment response separately (see Discussion). However, any potential influence of these variables was probed in a series of separate preliminary exploratory analyses using ANCOVA to compare all outcome variables between groups, including the covariates of baseline score, treatment duration, and days from baseline to post-intervention.

Analysis of covariance (ANCOVA; with baseline score as covariate) was the preferred method of primary analysis for all outcome variables (Egbewale et al., 2014; Kraemer, 2015; Overall \& Ashby, 1991), which were analysed separately. ANCOVA could not be applied to two analyses (Inhibition/Shifting vs. Colour Naming and Q-LES-Q-SF) due to non-normal distributions, as determined by Shapiro-Wilk tests of within-group and overall model standardized residuals. Subsequently, Quade's (1967) rank ANCOVA was conducted as a nonparametric ANCOVA alternative (Olejnik \& Algina, 1984). In a further two analyses (TMT and DERS), ANCOVA was deemed to be inappropriate due to violation of statistical 
assumptions. As a result, two 2 (Time: Pre- vs. Post-intervention) x 2 (Treatment: CR vs. TAU) mixed-design analyses of variance (ANOVAs) were conducted with a logarithmic data transformation for one variable (TMT) whereas the other (DERS) was not able to be transformed and is reported despite violation of the assumption of homogeneity of variances, as assessed by Levene's test of homogeneity of variance ( $p$ $<.05)$.

\section{Results}

Table 1 presents socio-demographic characteristics and substance use and head injury history for CR vs. TAU groups. There were no significant differences in age (CR: $M d n=32.5$ years, range 22-56; TAU: $\mathrm{Mdn}=33$ years, range 19-53), education (CR: $M d n=12$ years, range 7-15; TAU: $M d n=12$ years, range 7-20), intelligence, employment, marital status, or history of losing consciousness/concussion or hospitalisation after a head injury. Additionally, primary substance of misuse did not significantly differ between groups, with significant heterogeneity observed. The descriptive data regarding substance use history presented in Table 1 suggests that participants used a variety of substances over many years, with particularly high rates of polysubstance use. Treatment duration was significantly longer $(p=.003)$ for participants in the CR group (Mdn $=67$ days, range 16-160) compared to the TAU group ( $\mathrm{Mdn}=25$ days, range 2-82). Additionally, there was a 5-day difference between groups in the interval between baseline and post-intervention assessments $(p=.005)(\mathrm{CR}: \mathrm{Mdn}=34$ days, range 33-36; TAU: $\operatorname{Mdn}=29$ days, range 26-36).

\section{Table 1}


Baseline socio-demographic, substance use and head injury characteristics of residents of a femaleonly substance use therapeutic community

\begin{tabular}{|c|c|c|c|}
\hline Characteristic & $\begin{array}{l}\text { e Remediation } \\
(n=16)\end{array}$ & $\begin{array}{l}\text { Treatment As Usual } \\
\qquad(n=17)\end{array}$ & $p$ \\
\hline$\overline{\operatorname{Age}(M, S D)^{\mathrm{a}}}$ & $33.6(10.1)$ & $32.9(7.6)$ & .85 \\
\hline Education $(M, S D)^{\mathrm{a}}$ & $11.3(2.4)$ & $11.6(2.6)$ & .82 \\
\hline Test of Premorbid Functioning $(M, S D)$ & $96.0(11.7)$ & $99.1(12.9)$ & .48 \\
\hline Unemployed $(\%)^{\mathrm{b}}$ & 81.2 & 82.4 & 1.00 \\
\hline Marital status (\% single $)^{\mathrm{b}}$ & 75.0 & 82.4 & .69 \\
\hline Primary substance of misuse $(\%)^{\mathrm{c}}$ & & & .20 \\
\hline Methamphetamine & 50.0 & 29.4 & \\
\hline Alcohol & 18.8 & 35.3 & \\
\hline Amphetamines & 12.5 & 0 & \\
\hline Heroin & 6.2 & 11.8 & \\
\hline Cannabis & 0 & 17.6 & \\
\hline Sedatives & 12.5 & 5.9 & \\
\hline \multicolumn{4}{|l|}{ Years of regular use } \\
\hline Alcohol (any use; $M, M d n, S D ; n$ ) & $12.9(13.5,9.0 ; 12)$ & \multicolumn{2}{|c|}{$9.5(6.5,7.0 ; 16)$} \\
\hline Alcohol (to intoxication; $M, M d n, S D ; n$ ) & $10.0(8.0,7.2 ; 11)$ & \multicolumn{2}{|c|}{$8.0(5.0,7.0 ; 16)$} \\
\hline Heroin $(M, M d n, S D ; n)$ & $16.0(n=1)$ & \multicolumn{2}{|c|}{$9.7(8.0,8.6 ; 5)$} \\
\hline Methadone $(M, M d n, S D ; n)$ & $8.0(n=1)$ & \multicolumn{2}{|c|}{$8.3(8.3,11.0 ; 2)$} \\
\hline Other opiates/analgesics $(M, M d n, S D ; n)$ & $11.5(12.0,4.3 ; 3)$ & \multicolumn{2}{|c|}{$7.2(6.0,5.7 ; 5)$} \\
\hline Sedatives/hypnotics/tranquilizers $(M, M d n, S D ; n)$ & $10.6(12.0,4.4 ; 5)$ & \multicolumn{2}{|c|}{$6.3(3.5,6.0 ; 8)$} \\
\hline Cocaine $(M, M d n, S D ; n)$ & $7.0(4.0,7.9 ; 3)$ & \multicolumn{2}{|c|}{$7.0(8.5,4.2 ; 4)$} \\
\hline Amphetamines $(M, M d n, S D ; n)$ & $7.8(8.0,5.6 ; 14)$ & \multicolumn{2}{|c|}{$9.2(10.5,6.1 ; 12)$} \\
\hline Cannabis $(M, M d n, S D ; n)$ & $11.2(10.0,7.7 ; 13)$ & \multicolumn{2}{|c|}{$10.5(12.5,6.6 ; 13)$} \\
\hline Hallucinogens $(M, M d n, S D ; n)$ & $5.2(3.0,6.1 ; 5)$ & \multicolumn{2}{|c|}{$8.0(8.0,5.7 ; 2)$} \\
\hline More than one substance per day $(M, M d n, S D ; n)$ & $9.5(9.5,4.9 ; 12)$ & \multicolumn{2}{|c|}{$10.6(10.0,6.7 ; 15)$} \\
\hline Lost consciousness/concussion after head injury $(\%)^{\mathrm{c}}$ & 50.0 & 58.8 & .61 \\
\hline Hospitalized after head injury $(\%)^{\mathrm{c}}$ & 25.0 & 47.1 & .19 \\
\hline
\end{tabular}

${ }^{\text {a }}$ Mann-Whitney $U$ test. ${ }^{\text {b }}$ Fisher's exact test. ${ }^{\mathrm{c}}$ Chi-square test.

Psychiatric comorbidity data for the CR vs. TAU groups are displayed in Table 2. No significant differences between groups were found for the proportions meeting criteria for current or past Axis I diagnoses (other than SUDs), personality disorders, or the full range of other psychiatric comorbidities. 
Table 2

Psychiatric comorbidities of residents of a female-only substance use therapeutic community

\begin{tabular}{|c|c|c|c|}
\hline Psychiatric Comorbidity & $\begin{array}{l}\text { e Remediation } \\
(n=16)\end{array}$ & $\begin{array}{l}\text { Treatment As Usual } \\
\qquad(n=17)\end{array}$ & $p$ \\
\hline Current Axis I psychiatric diagnosis ${ }^{\mathrm{a}}(\%)$ & 56.2 & 76.5 & .22 \\
\hline Past Axis I psychiatric diagnosis ${ }^{\mathrm{a}}(\%)$ & 68.8 & 64.7 & .81 \\
\hline Personality disorder screen (met criteria) ${ }^{\mathrm{a}}(\%)$ & 50.0 & 41.2 & 61 \\
\hline Major depressive episode $^{a}$ & & & .81 \\
\hline Never (lifetime) & 50.0 & 35.3 & \\
\hline Major depressive episode (past 2 weeks) & 18.7 & 11.8 & \\
\hline Substance-induced mood disorder (past 2 weeks) & 0 & 5.9 & \\
\hline $\begin{array}{l}\text { Mood disorder due to medical condition } \\
\text { (past } 2 \text { weeks) }\end{array}$ & 0 & 5.9 & \\
\hline Past major depressive episode & 25.0 & 35.3 & \\
\hline Past mood disorder due to medical condition & 6.2 & 5.9 & \\
\hline Dysthymia $^{a}$ & & & .40 \\
\hline Never (lifetime) & 56.2 & 76.5 & \\
\hline Dysthymia (past 2 years) & 25.0 & 17.6 & \\
\hline Past dysthymia & 18.8 & 5.9 & \\
\hline Manic episode ${ }^{a}$ & & & .54 \\
\hline Never (lifetime) & 87.5 & 70.6 & \\
\hline Current manic episode & 0 & 0 & \\
\hline Past manic episode & 0 & 11.8 & \\
\hline Past hypomanic episode & 6.2 & 5.9 & \\
\hline Past substance-induced hypomanic episode & 6.2 & 5.9 & \\
\hline Past hypomanic episode due to medical condition & 0 & 5.9 & \\
\hline Panic disorder ${ }^{\mathrm{a}}$ & & & .63 \\
\hline Never (lifetime) & 62.5 & 70.6 & \\
\hline Panic disorder (past month) & 6.2 & 0 & \\
\hline \multicolumn{4}{|l|}{ Substance-induced anxiety disorder with } \\
\hline panic attacks (past month) & 0 & 5.9 & \\
\hline \multicolumn{4}{|l|}{ Anxiety disorder with panic attacks due to } \\
\hline a medical condition (past month) & 6.2 & 5.9 & \\
\hline Panic disorder (lifetime) & 18.8 & 5.9 & \\
\hline Panic disorder symptoms (lifetime) & 6.2 & 11.8 & \\
\hline Agoraphobia $^{\mathrm{a}}$ & & & .29 \\
\hline Never (lifetime) & 43.8 & 70.6 & \\
\hline Current agoraphobia & 31.2 & 17.6 & \\
\hline Agoraphobia (lifetime) & 25.0 & 11.8 & \\
\hline Social phobia ${ }^{\mathrm{b}}$ (past month) & & & 1.0 \\
\hline
\end{tabular}


Yes

Specific phobia ${ }^{\mathrm{b}}$ (past month)

No

100

Yes

Obsessive compulsive disorder

Not current (past month)

Posttraumatic stress disorder ${ }^{\mathrm{b}}$

Never (lifetime)

Posttraumatic stress disorder (past month)

Psychotic disorder ${ }^{\mathrm{b}}$ (lifetime)

Never

Substance-induced psychotic disorder

Anorexia nervosa

Not current (past 3 months)

100

Bulimia nervosa $^{\mathrm{b}}$ (past 3 months)

No

Yes

Generalized anxiety disorder ${ }^{\mathrm{a}}$ (past 6 months)

No

Yes

25.0

17.6

Substance-induced generalised anxiety disorder

Generalised anxiety disorder due to medical condition

Antisocial personality disorder ${ }^{\mathrm{b}}$ (lifetime)

Yes

Somatization disorder

Never (lifetime)

Hypochondriasis $^{\mathrm{b}}$

Hypochondriasis (past 6 months)

Pain disorder

Adult attention deficit/hyperactivity disorder ${ }^{\mathrm{b}}$ (lifetime)

Probable premenstrual dysphoric disorder ${ }^{\mathrm{b}}$ (past year) 
${ }^{\mathrm{a}}$ Chi-square test. ${ }^{\mathrm{b}}$ Fisher's exact test.

The exploratory ANCOVA analyses of all outcome variables including baseline score, treatment duration, and days from baseline to post-intervention as covariates, found treatment duration to be significant in only one analysis (BIS-11, $p$ $=.045)$ and the interval between baseline and post-intervention in only one other (BSCS, $p=.036$ ). These variables conferred only a small effect, with BIS-11 decreasing by .07 units for every one-day increase in treatment duration, and BSCS increasing by .06 units for every one-day increase in assessment interval.

Primary ANCOVA and mixed-design ANOVA results are displayed in Table 3. With baseline scores included as covariates, post-intervention scores in the CR group relative to the TAU group were significantly higher for the Color-Word Interference Test (Condition Three: Inhibition vs. Colour Naming) and significantly lower for GEC and MI of the BRIEF-A, with a trend also for BRI; a pattern of results indicating that the CR vs. TAU group displayed improvements in executive functions. The CR group also differed from the TAU group on the self-regulation measures, with lower BIS-11 and higher BSCS scores, as well as higher Q-LES-Q-SF scores, and a trend towards lower PACS scores. These results indicate that the CR group relative to the TAU group displayed reduced impulsivity, higher self-control, and higher quality of life, as well as a trend towards reduced cravings.

ANCOVA results indicated no significant differences between groups for WMI and Inhibition/Shifting vs. Color Naming, and mixed-design ANOVA results indicated no significant Time $\mathrm{x}$ Treatment interactions for TMT and the DERS. 
Table 3

Post-intervention effects of cognitive remediation vs. treatment as usual on executive functions, self-regulation and quality of life in residents of a female-only substance use therapeutic community

\begin{tabular}{|c|c|c|c|c|c|c|c|}
\hline \multirow[t]{3}{*}{ Dependent measures } & \multicolumn{2}{|c|}{$\begin{array}{l}\text { Cognitive Remediation (CR) } \\
(n=16)\end{array}$} & \multicolumn{2}{|c|}{$\begin{array}{l}\text { Treatment As Usual (TAU) } \\
(n=17)\end{array}$} & \multirow[t]{3}{*}{$F$} & \multirow[t]{3}{*}{$p$} & \multirow[t]{3}{*}{$\begin{array}{l}\text { Partial } \\
\eta^{2}\end{array}$} \\
\hline & Baseline & Post-intervention & Baseline & Post-intervention & & & \\
\hline & $M(S D)$ & Adjusted $M(S D)$ & $M(S D)$ & Adjusted $M(S D)$ & & & \\
\hline \multicolumn{8}{|l|}{ Executive functions } \\
\hline WMI & $91.88(10.61)$ & $96.97(6.08)$ & $93.53(13.01)$ & $95.85(6.08)$ & 0.28 & 0.60 & 0.009 \\
\hline Inhibition vs. Colour Naming & $9.44(3.01)$ & $11.44(1.73)$ & $10.47(2.00)$ & $10.18(1.73)$ & 4.29 & $0.047 *$ & 0.125 \\
\hline Inhibition/Shifting vs. Colour Naming ${ }^{\mathrm{a}}$ & $9.31(3.14)$ & $11.44(2.19)$ & $10.41(2.27)$ & $10.77(2.05)$ & 1.41 & 0.25 & 0.043 \\
\hline$\dagger \mathrm{TMT}(\mathrm{B}-\mathrm{A} ; \text { time s})^{\mathrm{b}, \mathrm{c}}$ & $38.47(17.63)$ & $35.27(20.79)$ & $31.50(9.27)$ & $21.13(7.78)$ & 1.80 & 0.19 & 0.058 \\
\hline \multicolumn{8}{|l|}{ BRIEF-A } \\
\hline †Global Executive Composite & $59.44(11.19)$ & $53.07(6.94)$ & $67.59(12.47)$ & $59.35(6.93)$ & 6.38 & $0.017^{*}$ & 0.175 \\
\hline$\dagger$ Behavioral Regulation Index & $60.63(12.40)$ & $55.28(7.74)$ & $67.59(10.24)$ & $60.32(7.73)$ & 3.34 & 0.08 & 0.100 \\
\hline$\dagger$ Metacognition Index & $57.31(10.36)$ & $51.46(6.50)$ & $66.41(13.78)$ & $57.04(6.49)$ & 5.69 & $0.024^{*}$ & 0.160 \\
\hline \multicolumn{8}{|l|}{ Self-regulation } \\
\hline$\dagger \mathrm{BIS}-11$ & $75.44(12.15)$ & 70.57 (7.39) & $77.76(14.08)$ & $76.11(7.39)$ & 4.61 & $0.04 *$ & 0.133 \\
\hline $\mathrm{BSCS}^{\mathrm{d}}$ & $2.81(0.90)$ & $2.90(0.42)$ & $2.13(0.87)$ & $2.55(0.40)$ & 5.53 & $0.026^{*}$ & 0.160 \\
\hline$\dagger \mathrm{DERS}^{\mathrm{b}, \mathrm{d}, \mathrm{e}}$ & $94.93(33.15)$ & $88.60(31.10)$ & $94.47(16.37)$ & $92.47(14.02)$ & 0.44 & 0.51 & 0.014 \\
\hline$\dagger \mathrm{PACS}^{\mathrm{f}}$ & $9.31(8.47)$ & $7.84(5.54)$ & $11.56(6.01)$ & $11.48(5.54)$ & 3.41 & 0.08 & 0.105 \\
\hline \multicolumn{8}{|l|}{ Quality of life } \\
\hline Q-LES-Q-SF ${ }^{\mathrm{a}, \mathrm{d}}$ & $0.62(0.12)$ & $0.71(0.06)$ & $0.55(0.18)$ & $0.61(0.16)$ & 7.68 & $0.01 * *$ & 0.204 \\
\hline
\end{tabular}

$* p \leq .05 * * p \leq .01$ 
ANCOVA analyses: Post-intervention adjusted means determined using baseline values as covariates.

Partial $\eta^{2}$ effect size interpretation guidelines (Cohen, 1988): $0.01=$ small; $0.06=$ medium; $0.14=$ large.

$\dagger$ Lower scores reflect better performance.

a Quade's nonparametric rank analysis of covariance: post-intervention unadjusted $M(S D)$, Quade's $F$ statistic.

${ }^{\mathrm{b}} 2$ × 2 mixed-design analysis of variance: post-intervention unadjusted $M(S D), F$-Interaction.

${ }^{\mathrm{c}}$ Logarithmic data transformation ( $n=31$ as 2 cases unable to be transformed).

${ }^{\mathrm{d}}$ CR group $n=15$.

${ }^{\mathrm{e}}$ Data could not be transformed.

${ }^{\mathrm{f}}$ TAU group $n=16$. 


\section{Discussion}

This study aimed to test the effectiveness of cognitive remediation to improve executive functions, self-regulation and quality of life in female residents of an SUD therapeutic community. It was based on a theoretical framework positing that basic EFs underpin effective self-regulation (i.e., the capacity for effective goal-directed behavior in everyday life; Hofmann et al., 2012). Results indicated that the group receiving the $\mathrm{CR}$ intervention improved performance on an inhibition task, facets of self-regulation (i.e., impulsivity and self-control) and quality of life post-intervention relative to the TAU group. CR also improved self-reported EFs (i.e., GEC and MI of the BRIEF-A, with a trend towards BRI) compared to TAU. Additionally, there was a trend towards reduced cravings in the CR vs. TAU group. These results provide significant new evidence for the potential utility of neuropsychological interventions in SUD treatment contexts. Importantly, the current study suggests that CR may be a viable intervention in SUD treatment settings where high rates of comorbidities such as past head injuries and psychiatric diagnoses are common.

Inhibition is one of the EFs recognized as making a significant contribution to the development and maintenance of SUD (Jentsch \& Pennington, 2014). The core inhibitory deficits contributing to SUD are increased tendencies to approach substances and/or the inability to suppress this approach-behavior, which manifest at different levels of analysis and have been assessed via behavioral task-specific and trait self-report methods (Gullo et al., 2014). There is ongoing debate over the use of self-report vs. behavioral laboratory task assessment methodology, with strengths and weakness of each method noted (Cyders \& Coskunpinar, 2011). The current study utilized task-specific assessment of inhibition and inventory-based measurement of impulsivity (i.e., BIS-11), with improvements in task-performance and reduced levels 
of trait impulsivity reported in the CR group. Although these findings may seem to converge, it has been noted that self-report measures vs. behavioral laboratory tasks may assess divergent components of the construct (Cyders \& Coskunpinar, 2011).

Self-control is inversely related to trait impulsivity and refers to the capacity to overcome unwanted urges to achieve goal-directed behavior (Hofmann et al., 2012). Self-control plays an important role in SUD (Volkow et al., 2013) and interacts dynamically with cravings in contributing to problematic substance use (Grasman et al., 2016). Decreased self-control is associated with cumulative stress and this relationship is moderated by self-reported, but not behavioral, impulsivity (Hamilton et al., 2014). The CR group in the current study reported increased self-control, which may have played a role in the marginal decrease also reported for cravings. Interventions that bolster self-control in SUD populations, such as CR, may help to shield against the deleterious effects of cravings (Fatseas et al., 2015) and stress (Sinha et al., 2011) in relapse to substance use.

Unlike previous cognitive remediation interventions that focused on one component of cognition (e.g., working memory training in Houben et al., 2011), the current study incorporated training exercises that involved a range of cognitive skills (e.g., working memory, selective attention, divided attention, planning, inhibition, and flexibility). As such, the reduced total training time on some components may partly explain the absence of effects on some EF domains. It is possible that the domains that showed gains had more time dedicated to them during the strategy and drill practice components of the program.

The CR group also demonstrated improvements in EFs as measured by the BRIEF-A. Specifically, these improvements were observed in the overall summary score (GEC) and one of the index scores (MI), with a trend towards improvement in 
the other index score (BRI). Given the minimal correlation between inventory- and performance-based measures of EFs and likelihood that such methods of assessment may be capturing distinct components of EFs (Toplak et al., 2013), the improvements observed across both types of measures in the current study may indicate a robust change in EF. Further research, however, is needed to tease apart the complex relationship between various components of EFs and self-regulation. This endeavour could involve diverse methodologies and several levels of analysis (e.g., self-report, laboratory tasks, neuropsychological assessment, neuroimaging techniques, clinical outcomes).

Quality of life spans broad areas of psychological wellbeing, physical health, level of independence, and social connectedness, and has been defined as "an individual's perception of their position in life in the context of the culture and value systems in which they live and in relation to their goals, expectations, standards and concerns" (Feelemyer et al., 2014, p. 251). Findings from the current study showed that CR impacted positively upon quality of life. This is a promising preliminary finding as quality of life is an important predictor of sustained remission from SUD (Laudet et al., 2009).

A recent study found that cognitive training improved EFs (i.e., working memory and reflection-impulsivity/decision-making) in polysubstance users in Therapeutic Community treatment (Valls-Serrano et al., 2016a). Importantly, that study was the first to also demonstrate improved self-regulation performance in an ecologically valid task of goal-directed behavior, the Multiple Errands Test contextualized version (Shallice \& Burgess, 1991; Valls-Serrano et al., 2016b). The study excluded participants with psychiatric comorbidities and those with a history of head injury to capture efficacy of cognitive training. In a previous study we 
demonstrated that $50 \%$ of residents in residential treatment for SUD had been hospitalized after sustaining a head injury and this was a significant determinant of cognitive impairment (Marceau et al., 2016). Psychiatric comorbidities are common (Baingana et al., 2015), with Borderline Personality Disorder diagnosed in up to $65 \%$ of individuals with SUDs (Pennay et al., 2011). Similarly, the current study found that over $50 \%$ of the overall sample met criteria for a current Axis I diagnosis, while over $40 \%$ had a positive screen for personality disorder. Over $50 \%$ of the sample had lost consciousness or been concussed after a head injury and over $30 \%$ had been hospitalized after a head injury. The current study extends the findings of VallsSerrano and colleagues (2016a) to a research context high in ecological validity by including participants with psychiatric or head injury comorbidities. In the present study, there were improvements on conventional neuropsychological tests (e.g., inhibition task), whereas that was not the case in the Valls-Serrano (2016a) study. This may be due to the inclusion of computerized cognitive training and/or because the present sample were more impaired by virtue of greater comorbidities. These preliminary results suggest that cognitive remediation may be a viable option to improve EFs and self-regulation for SUD populations, including those with psychiatric and/or head injury comorbidities.

A strength of the current study is that it shows significant promise for the use of CR interventions as adjunct treatments for SUD. To the best of our knowledge, this is the first study to inclusively demonstrate feasibility and effectiveness of neuropsychological intervention for female residents that engage in SUD therapeutic communities, without exclusion of those with psychiatric and head injury comorbidities. Certain limitations, however, must be noted. The sample size of the current study was small and it used a controlled sequential groups design. Related to 
the constraints of this design, the significant difference in length of treatment and assessment interval between groups was not ideal. This is particularly relevant to the observed improvements in impulsivity and self-control, as these improvements were, to some degree, respectively related to variance in treatment length and assessment interval observed between groups. Future studies should investigate the benefit of CR in groups matched on interval between assessments and treatment duration, accounting for the heterogeneity of treatment length occurring across SUD populations in residential facilities. Specifically, studies should further examine the role of treatment and/or abstinence length in relation to the neurocognitive and selfregulation improvements that may occur as a result of cognitive training. It is also important to note that while statistical tests revealed no significant differences in socio-demographic, psychiatric, head injury, and primary substance of misuse characteristics between CR vs. TAU groups, there were apparent differences in history of substance use (i.e., years of regular use across substances) which were not compared statistically due to insufficient subgroup sample sizes. Taken together, and particularly in light of the small sample size of this study, these limitations raise the possibility of significant between-group variability due to the range of potential confounding factors (e.g., treatment duration and hence abstinence length, assessment interval, primary substance of misuse, and substance use history). For these reasons, it is imperative that future studies aim to replicate the current findings in larger, multisite, randomized controlled trials, with the inclusion of a well-matched, active control group. It is hoped that this study prompts much-needed further research that may aim to replicate these findings and more thoroughly investigate the effectiveness of cognitive training in SUD populations with comorbid conditions such as psychiatric diagnoses and history of head injury. 
Rates of cognitive recovery across the first year of SUD treatment have been investigated at group and individual levels, with qualitatively different trajectories of change observed between individuals (Bates et al., 2013b). This has implications for the development and implementation of neuropsychological interventions in SUD treatment contexts. As this area of research grows, studies could begin to explore the effectiveness of interventions for SUD across varying subgroups, in order to ascertain the influence of individual differences that are present in the often heterogeneous makeup of SUD populations, and tailor interventions accordingly. Studies that also track clinical outcomes related to sustained remission from SUDs (e.g., quality of life, length of stay in treatment, program completion, dropout, relapse to substance use) will be beneficial in further informing neuropsychological interventions as a strategy to reduce the individual and collective harms associated with SUDs. Nevertheless, the preliminary evidence from the current study suggests that CR may improve EFs, selfregulation, and quality of life, and with further examination may be a promising intervention for use within residential SUD treatment settings. 


\section{References}

Alfonso, J., Caracuel, A., Delgado-Pastor, L., Verdejo-García, A., 2011. Combined goal management training and mindfulness meditation improve executive functions and decision-making performance in abstinent polysubstance abusers. Drug Alcohol Depen. 117, 78-81. doi:10.1016/j.drugalcdep.2010.12.025

American Psychiatric Association, 2000. Diagnostic and Statistical Manual of Mental Disorders, fourth ed., text rev. Author, Washington, D.C.

Baingana, F., al'Absi, M., Becker, A.E., Pringle, B., 2015. Global research challenges and opportunities for mental health and substance-use disorders. Nature 527, S172-S177. doi:10.1038/nature16032

Barkley, R.A., 2001. The executive functions and self-regulation: An evolutionary neuropsychological perspective. Neuropsychol. Rev. 11, 1-29.

Barkley, R.A., 2012. Executive Functions: What They Are, How They Work, and Why They Evolved. Guilford Press, New York.

Bates, M.E., Buckman, J.F., Nguyen, T.T, 2013a. A role for cognitive rehabilitation in increasing the effectiveness of treatment for alcohol use disorders. Neuropsychol. Rev. 23, 27-47. doi:10.1007/s11065-013-9228-3

Bates, M.E., Buckman, J.F., Eddie, D., Freeman, J., Voelbel, G.T., 2013b. The mean and the individual: Integrating variable-centered and person-centered analyses of cognitive recovery in patients with substance use disorders. Front. Psychiatry 4, 1-12. doi:10.3389/fpsyt.2013.00177

Bechara, A., Damasio, A.R., Damasio, H., \& Anderson, S.W., 1994. Insensitivity to future consequences following damage to human prefrontal cortex. Cognition 50, 7-15. doi:10.1016/0010-0277(94)90018-3 
Bickel, W.K., Yi, R., Landes, R.D., Hill, P.F., Baxter, C., 2011. Remember the future: Working memory training decreases delay discounting among stimulant addicts. Biol. Psychiatry 69, 260-265. doi:10.1016/j.biopsych.2010.08.017

Brorson, H.H., Ajo Arnevik, E., Rand-Hendriksen, K., Duckert, F., 2013. Drop-out from addiction treatment: A systematic review of risk factors. Clin. Psychol. Rev. 33, 1010-1024. doi:10.1016/j.cpr.2013.07.007

Charvet, L. E., Shaw, M. T., Haider, L., Melville, P., Krupp, L. B., 2015. Remotelydelivered cognitive remediation in multiple sclerosis (MS): Protocol and results from a pilot study. Mult. Scler. J. Exp. Transl. Clin. 1, 1-10. doi:10.1177/2055217315609629

Cohen, J., 1988. Statistical Power Analysis for the Behavioural Sciences, second ed. Academic Press, New York.

Cyders, M.A., Coskunpinar, A., 2011. Measurement of constructs using selfreport and behavioral lab tasks: Is there overlap in nomothetic span and construct representation for impulsivity? Clin. Psychol. Rev. 31, 965-982. doi:10.1016/j.cpr.2011.06.001

Dannhauser, T. M., Cleverley, M., Whitfield, T. J., Fletcher, B., Stevens, T., Walker, Z., 2014. A complex multimodal activity intervention to reduce the risk of dementia in mild cognitive impairment-ThinkingFit: Pilot and feasibility study for a randomized controlled trial. BMC Psychiatry 14. doi: 10.1186/1471244X-14-129

Darke, S., Campbell, G., Popple, G., 2012. Retention, early dropout and treatment completion among therapeutic community admissions. Drug Alcohol Rev. 31, 64-71. doi:10.1111/j.1465-3362.2011.00298.x 
Delis, D.C., Kaplan, E., Kramer, J.H., 2001. Delis-Kaplan Executive Function System: D-KEFS. The Psychological Corporation, San Antonio; Texas.

Duckworth, A. L., Grant, H., Loew, B., Oettingen, G., Gollwitzer, P. M., 2011. Selfregulation strategies improve self-discipline in adolescents: Benefits of mental contrasting and implementation intentions. Educ. Psychol.-UK. 31, 17-26. doi:10.1080/01443410.2010.506003

Egbewale, B.E., Lewis, M., Sim, J., 2014. Bias, precision and statistical power of analysis of covariance in the analysis of randomized trials with baseline imbalance: A simulation study. BMC Med. Res. Methodol. 14. doi:10.1186/1471-2288-14-49

Endicott, J., Nee, J., Harrison, W., Blumenthal, R., 1993. Quality of Life Enjoyment and Satisfaction Questionnaire: A new measure. Psychopharmacol. Bull. 29, $321-326$.

Fasotti, L., Kovacs, F., Eling, P. A. T. M., Brouwer, W. H., 2000. Time pressure management as a compensatory strategy training after closed head injury. Neuropsychol. Rehabil. 10, 47-65.

Fatseas, M., Serre, F., Alexandre, J., Debrabant, R., Auriacombe, M., Swendsen, J., 2015. Craving and substance use among patients with alcohol, tobacco, cannabis or heroin addiction: A comparison of substance- and person-specific cues. Addiction 110, 1035-1042. doi:10.1111/add.12882

Feelemyer, J.P., Des Jarlais, D.C., Arasteh, K., Phillips, B.W., Hagan, H., 2014. Changes in quality of life (WHOQOL-BREF) and addiction severity index (ASI) among participants in opioid substitution treatment (OST) in low and middle income countries: An international systematic review. Drug Alcohol Depend. 134, 251-258. doi:10.1016/j.drugalcdep.2013.10.011 
Fernández-Serrano, M., Pérez-García, M., Schmidt Río-Valle, J., Verdejo-García, A., 2010. Neuropsychological consequences of alcohol and drug abuse on different components of executive functions. J. Psychopharmacol. 24, 13171332. doi:10.1177/0269881109349841

Fertuck, E.A., Lenzenweger, M.F., Clarkin, J.F., Hoermann, S., Stanley, B., 2006. Executive neurocognition, memory systems, and borderline personality disorder. Clin. Psychol. Rev. 26, 346-375. doi:10.1016/j.cpr.2005.05.008

Flannery, B.A., Volpicelli, J.R., Pettinati, H.M., 1999. Psychometric properties of the Penn Alcohol Craving Scale. Alcohol Clin. Exp. Res. 23, 1289-1295.

Friedman, N.P., Miyake, A., 2016. Unity and diversity of executive functions: Individual differences as a window on cognitive structure. Cortex doi:10.1016/j.cortex.2016.04.023

Golden, C.J., 1978. Stroop color and word test. Stoelting Company, Wood Dale; Illinois.

Gonzalez, C., 2014. Screening for personality disorder in drug and alcohol dependence. Psychiat. Res. 217, 121-123. doi:10.1016/j.psychres.2014.03.007

Grasman, J., Grasman, R.P., van der Maas, H.J., 2016. The dynamics of addiction: Craving versus self-control. PLoS ONE 11, 1-11. doi:10.1371/journal.pone.0158323

Gratz, K.L., Roemer, L., 2004. Multidimensional assessment of emotion regulation and dysregulation: Development, factor structure, and initial validation of the Difficulties in Emotion Regulation Scale. J. Psychopathol. Behav. Assess. 26, $41-54$. 
Gullo, M.J., Loxton, N.J., Dawe, S., 2014. Impulsivity: Four ways five factors are not basic to addiction. Addict. Behav. 39, 1547-1556. doi:10.1016/j.addbeh.2014.01.002

Hagen, E., Erga, A.H., Hagen, K.P., Nesvåg, S.M., McKay, J.R., Lundervold, A.J., Walderhaug, E., 2016. Assessment of executive function in patients with substance use disorder: A comparison of inventory- and performance-based assessment. J. Subst. Abuse Treat. 66, 1-8. doi:10.1016/j.jsat.2016.02.010

Hamilton, K.R., Sinha, R., Potenza, M.N., 2014. Self-reported impulsivity, but not behavioral approach or inhibition, mediates the relationship between stress and self-control. Addict. Behav. 39, 1557-1564. doi:10.1016/j.addbeh.2014.01.003

Heatherton, T.F., Wagner, D.D., 2011. Cognitive neuroscience of self-regulation failure. Trends Cogn. Sci. 15, 132-139. doi:10.1016/j.tics.2010.12.005

Hesse, M., Moran, P., 2010. Screening for personality disorder with the Standardised Assessment of Personality: Abbreviated Scale (SAPAS): Further evidence of concurrent validity. BMC Psychiatry 10. doi:10.1186/1471-244x-10-10

Hesse, M., Rasmussen, J., Pedersen, M.K., 2008. Standardised assessment of personality: A study of validity and reliability in substance abusers. BMC Psychiatry 8. doi:10.1186/1471-244X-8-7

Hester, R., Lubman, D. I., Yücel, M., 2010. The role of executive control in human drug addiction. Curr. Top. Behav. Neurosci. 3, 301-318. doi:10.1007/7854_2009_28

Hofmann, W., Schmeichel, B.J., Baddeley, A.D., 2012. Executive functions and selfregulation. Trends Cogn. Sci. 16, 174-180. doi:10.1016/j.tics.2012.01.006 
Houben, K., Wiers, R.W., Jansen, A., 2011. Getting a grip on drinking behavior: Training working memory to reduce alcohol abuse. Psychol. Sci. 22, 968-975.

IBM Corp, 2012. IBM SPSS Statistics for Windows, Version 21.0. Author, Armonk; New York.

Isquith, P.K., Roth, R.M., Gioia, G., 2013. Contribution of rating scales to the assessment of executive functions. Appl. Neuropsychol. Child. 2, 125-132. doi:10.1080/21622965.2013.748389

Jentsch, J.D., Pennington, Z.T., 2014. Reward, interrupted: Inhibitory control and its relevance to addictions. Neuropharmacology 76, 479-486. doi:10.1016/j.neuropharm.2013.05.022

Jurado, M., Rosselli, M., 2007. The elusive nature of executive functions: A review of our current understanding. Neuropsychol. Rev. 17, 213-233. doi:10.1007/s11065-007-9040-z

Kesler, S. R., Lacayo, N. J., Jo, B., 2011. A pilot study of an online cognitive rehabilitation program for executive function skills in children with cancerrelated brain injury. Brain Inj. 25, 101-112. doi:10.3109/02699052.2010.536194

Kraemer, H.C., 2015. A source of false findings in published research studies: Adjusting for covariates. JAMA Psychiatry 72, 961-962. doi:10.1001/jamapsychiatry.2015.1178

Laudet, A.B., Becker, J.B., White, W.L., 2009. Don't wanna go through that madness no more: Quality of life satisfaction as predictor of sustained remission from illicit drug misuse. Subst. Use Misuse 44, 227-252. doi:10.1080/10826080802714462 
Levine, B., Schweizer, T. A., O'Connor, C., Turner, G., Gillingham, S., Stuss, D. T., . . Robertson, I. H., 2011. Rehabilitation of executive functioning in patients with frontal lobe brain damage with goal management training. Front. Hum. Neurosci. 5. doi:10.3389/fnhum.2011.00009

Lezak, M.D., Howieson, D.B., Bigler, E.D., Tranel, D., 2012. Neuropsychological Assessment, fifth ed. Oxford University Press, New York.

Lumosity, 2016. Lumosity. Retrieved September 20, 2016, from https://www.lumosity.com/

Marceau, E.M., Lunn, J., Berry, J., Kelly, P.J., Solowij, N., 2016. The Montreal Cognitive Assessment (MoCA) is sensitive to head injury and cognitive impairment in a residential alcohol and other drug therapeutic community. J. Subst. Abuse Treat. 66, 30-36. doi:10.1016/j.jsat.2016.03.002

McLellan, A.T., Kushner, H., Metzger, D., Peters, R., Smith, I., Grissom, G., Pettinati, H., Argeriou, M., 1992. The fifth edition of the Addiction Severity Index. J. Subst. Abuse Treat. 9, 199-213. doi:10.1016/0740-5472(92)90062-S

Miyake, A., Friedman, N.P., Emerson, M.J., Witzki, A.H., Howerter, A., Wager, T.D., 2000. The unity and diversity of executive functions and their contributions to complex "frontal lobe" tasks: A latent variable analysis. Cognitive Psychol. 41, 49-100. doi:10.1006/cogp.1999.0734

Moran, P., Leese, M., Lee, T., Walters, P., Thornicroft, G., Mann, A., 2003. Standardised Assessment of Personality - Abbreviated Scale (SAPAS): Preliminary validation of a brief screen for personality disorder. Brit. J. Psychiat. 183, 228-232.

O’Connell, R. G., Bellgrove, M. A., Dockree, P. M., Lau, A., Fitzgerald, M., Robertson, I. H., 2008. Self-alert training: Volitional modulation of autonomic 
arousal improves sustained attention. Neuropsychologia 46, 1379-1390. doi:10.1016/j.neuropsychologia.2007.12.018

Olejnik, S.F., Algina, J., 1984. A review of nonparametric alternatives to analysis of covariance. Evaluation Rev. 9, 239-257.

Overall, J.E., Ashby B., 1991. Baseline corrections in experimental and quasiexperimental clinical trials. Neuropsychopharmacology 4, 273-281.

Paquin, K., Larouche Wilson, A., Cellard, C., Lecomte, T., Potvin, S., 2014. A systematic review on improving cognition in schizophrenia: Which is the more commonly used type of training, practice or strategy learning? BMC Psychiatry 14, 1-32. doi:10.1186/1471-244X-14-139

Patton, J.H., Stanford, M.S., Barratt, E.S., 1995. Factor structure of the Barratt Impulsiveness Scale. J. Clin. Psychol. 51, 768-774. doi:10.1002/10974679(199511)51:6<768::AID-JCLP2270510607>3.0.CO;2-1

Pennay, A., Cameron, J., Reichert, T., Strickland, H., Lee, N.K., Hall, K., Lubman, D.I., 2011. A systematic review of interventions for co-occurring substance use disorder and borderline personality disorder. J. Subst. Abuse Treat. 41, 363-373. doi:10.1016/j.jsat.2011.05.004

Pearson Assessment, 2009. Advanced Clinical Solutions for WAIS-IV and WMS-IV: Clinical and Interpretive Manual. NCS Pearson Inc., San Antonio; Texas.

Quade, D., 1967. Rank analysis of covariance. J. Am. Stat. Assoc. 62, 1187-1200.

Robertson, I. H., Tegnér, R., Tham, K., Lo, A., Nimmo-Smith, I., 1995. Sustained attention training for unilateral neglect: Theoretical and rehabilitation implications. J. Clin. Exp. Neuropsychol. 17, 416-430. doi:10.1080/01688639508405133

Roth, R.M., Isquith, P.K., Gioia, G.A., 2005. Behavioral Rating 
Inventory of Executive Function - Adult Version. Psychological Assessment Resources Inc., Lutz; Florida.

Ruocco, A.C., \& Carcone, D., 2016. A neurobiological model of borderline personality disorder: Systematic and integrative review. Harv. Rev. Psychiatry 24, 311-329. doi:10.1097/HRP.0000000000000123

Shallice, T., Burgess, P.W., 1991. Deficits in strategy application following frontal lobe damage in man. Brain J. Neurol. 114, 727-741.

Sheehan, D.V., Lecrubier, Y., Sheehan, K.H., Amorim, P., Janavs, J., Weiller, E., Hergueta, T., Baker, R., Dunbar, G.C., 1998. The Mini-International Neuropsychiatric Interview (M.I.N.I): The development and validation of a structured diagnostic psychiatric interview for DSM-IV and ICD-10. J. Clin. Psychiat. 59 (Suppl. 20), 22-33.

Sinha, R., Shaham, Y., Heilig, M., 2011. Translational and reverse translational research on the role of stress in drug craving and relapse. Psychopharmacology 218, 69-82. doi:10.1007/s00213-011-2263-y

Spikman, J. M., Boelen, D. H. E., Lamberts, K. F., Brouwer, W. H., Fasotti, L., 2010. Effects of a multifaceted treatment program for executive dysfunction after acquired brain injury on indications of executive functioning in daily life. J. Int. Neuropsychol. Soc. 16, 118-129. doi:10.1017/S1355617709991020

Strauss, E., Spreen, O., Sherman, E.S., 2006. A Compendium of Neuropsychological Tests: Administration, Norms, and Commentary, third ed. Oxford University Press, New York.

Tangney, J.P., Baumeister, R.F., Boone, A.L., 2004. High self-control predicts good adjustment, less pathology, better grades, and interpersonal success. J. Pers. 72, 271-324. doi:10.1111/j.0022-3506.2004.00263.x 
Toplak, M. E., West, R. F., Stanovich, K. E., 2013. Practitioner review: Do performance-based measures and ratings of executive function assess the same construct? J. Child Psychol. Psychiatry 54, 131-143. doi:10.1111/jcpp.12001

Unoka, Z., \& Richman, M.J., 2016. Neuropsychological deficits in BPD patients and the moderator effects of co-occurring mental disorders: A meta-analysis. Clin. Psychol. Rev. 44, 1-12. doi:10.1016/j.cpr.2015.11.009

Valls-Serrano, C., Caracuel, A., Verdejo-García, A., 2016a. Goal management training and mindfulness meditation improves executive functions and transfers to ecological tasks of daily life in polysubstance users enrolled in therapeutic community treatment. Drug Alcohol Depen. 165, 9-14. doi:10.1016/j.drugalcdep.2016.04.040

Valls-Serrano, C., Verdejo-García, A., Caracuel, A., 2016b. Planning deficits in polysubstance dependent users: Differential associations with severity of drug use and intelligence. Drug Alcohol Depend. 162, 72-78.

doi:10.1016/j.drugalcdep.2016.02.027

Vanderplasschen, W., Colpaert, K., Autrique, M., Rapp, R.C., Pearce, S., Broekaert, E., Vandevelde, S., 2013. Therapeutic communities for addictions: A review of their effectiveness from a recovery-oriented perspective. Sci. World J. 2013, 1-22. doi:10.1155/2013/427817

Volkow, N.D., Wang, G., Tomasi, D., Baler, R.D., 2013. Obesity and addiction: Neurobiological overlaps. Obes. Rev. 14, 2-18. doi:10.1111/j.1467-789X.2012.01031.X

Wechsler, D., 2008. Wechsler Adult Intelligence Scale, fourth ed. Pearson, Sydney; Australia. 
Wykes, T., Huddy, V., Cellard, C., McGurk, S., Czobor, P., 2011. A meta-analysis of cognitive remediation for schizophrenia: Methodology and effect sizes. Am. J. Psychiatry 168, 472-485. 